\title{
Polarization-based Inverse Rendering from a Single View
}

\author{
Daisuke Miyazaki* \\ Robby T. Tan* \\ Kenji $\mathrm{Hara}^{\dagger}$ \\ Katsushi Ikeuchi* \\ The University of Tokyo, Japan \\ http://www.cvl.iis.u-tokyo.ac.jp/
}

\begin{abstract}
This paper presents a method to estimate geometrical, photometrical, and environmental information of a singleviewed object in one integrated framework under fixed viewing position and fixed illumination direction. These three types of information are important to render a photorealistic image of a real object. Photometrical information represents the texture and the surface roughness of an object, while geometrical and environmental information represent the $3 D$ shape of an object and the illumination distribution, respectively. The proposed method estimates the $3 D$ shape by computing the surface normal from polarization data, calculates the texture of the object from the diffuse only reflection component, determines the illumination directions from the position of the brightest intensity in the specular reflection component, and finally computes the surface roughness of the object by using the estimated illumination distribution.
\end{abstract}

\section{Introduction}

It is well known that to successfully render a photorealistic image of a real object, one should have the information of object's physical information and its environment. Principally, three major types of information are requisite, i.e., geometrical, photometrical, and environmental information. Photometrical information provides the surface reflectance parameters (= texture and surface roughness) of an object, while geometrical and environmental information provides the $3 \mathrm{D}$ shape of an object and the illumination distribution, respectively.

Rendering this three types of information under less constraints are called inverse rendering. Sato et al. [1] and Tominaga et al. [2] estimated shape, texture, and surface roughness. Zheng et al. [3], Nayar et al. [4], and Kim et al. [5] estimated shape, texture, and illumination distribution. Ikeuchi et al. [6], Sato et al. [7], Ramamoorthi et al. [8], Nishino et al. [9], and Hara et al. [10,11] estimated

\footnotetext{
* Department of Computer Science, Graduate School of Information Science and Technology

${ }^{\dagger}$ Fukuoka Industrial Technology Center

‡ This research was supported in part by Japan Science and Technology Corporation under the Ikeuchi CREST project.
}

texture, surface roughness, and illumination distribution.

In this paper, we propose a novel method to obtain shape, texture, surface roughness, and illumination distribution from a single view in one integrated framework, with different approach from Rahmann's method [12]. The threedimensional shape of the target object is obtained by computing the surface normal from polarization data. The texture is calculated from the diffuse only reflection component, after separating the reflection components of the images based on color. The illumination directions are determined from the position of the brightest intensity in the specular component. Finally, the surface roughness of the object is computed by using the estimated illumination distribution.

The rest of this paper is organized as follows: Section 2 shows a method to obtaining the surface shape of the object. Section 3 shows the method to estimate the texture, surface roughness, and illumination direction. Section 4 shows the implemented algorithm. Section 5 shows the measurement results and Section 6 concludes this paper.

\section{Modeling geometric information}

Three-dimensional shape of the object is computed from images taken by a polarizer-mounted camera. We apply a method described in Section 2.1 to separate the reflection components before analyzing the polarization state of the input images. Section 2.2 describes a technique to compute the surface normal of the object by analyzing the polarization data of the diffuse reflection component. Surface height is estimated from the distribution of the surface normal by using a relaxation method $[13,14]$.

\subsection{Separating diffuse and specular components}

Most inhomogeneous surfaces follow the dichromatic reflection model. According to this model, the intensity of the light reflected from an object surface is a linear combination of the diffuse and the specular reflection components [15]. In our parameters estimation method, we need to separate the input image into two component images, namely, specular component image and diffuse component image.

Many methods have been proposed to separate the image into each components based on the color analysis. In our 
method, Tan's method [16] is used for its robustness. This method requires illumination chromaticity normalization, so that the illumination chromaticity needs to be known beforehand; we can use either white reference or color constancy algorithm to obtain the illumination chromaticity [17]. The normalization is done simply by dividing the image intensity using known illumination chromaticity: $\tilde{\mathbf{I}}=$ $\left[\begin{array}{lll}I_{R} / i_{r} & I_{G} / i_{g} & I_{B} / i_{b}\end{array}\right]^{T}$, where $\mathbf{I}=\left[\begin{array}{lll}I_{R} & I_{G} & I_{B}\end{array}\right]^{T}$ is the image intensity in each color channel, and $\mathbf{i}=\left[\begin{array}{lll}i_{r} & i_{g} & i_{b}\end{array}\right]^{T}$ is the illumination chromaticity. In this paper, we assume that all light sources have the same color throughout the surface.

\subsection{Polarization}

Shape-from-polarization [18-23] estimate the surface normal of an object from a single view illuminated by incoherent light sources, even without knowing any illumination information such as intensity, color, shape, and direction. The polarization state of diffuse light as well as specular light depends on the surface normal of the object, thus, by analyzing the polarization state of diffuse light, we can obtain the information about the shape of the object. By observing the light by a linear-polarizer-mounted camera, we can obtain the polarization state of the light. The light intensity will vary when the polarizer is rotated. This intensity difference forms a sinusoidal curve with respect to the angle of the rotation of the polarizer. We denote the maximum intensity of such sinusoidal curve as $I_{\max }$ and the minumum one as $I_{\min }$.

Surface normal can be represented as polar coordinate system $(\theta, \phi)$ where $\theta$ is zenith angle and $\phi$ is azimuth angle $\left(0^{\circ} \leq \theta<90^{\circ}, 0^{\circ} \leq \phi<360^{\circ}\right)$. Here, we set the camera at $\theta=0^{\circ}$. Zenith angle is the angle between the viewing direction and the surface normal, and azimuth angle is the orientation of the plane consisted of viewing direction and the surface normal.

The angle of the polarizer where we observe $I_{\max }$ will be the azimuth angle [18-23]. Since the linear polarizer has a cycle of $180^{\circ}$, we obtain two azimuth angles in the domain $0^{\circ} \leq \phi<360^{\circ}$. One of those angles will equal to the true azimuth angle of the surface normal, while the other will face to the opposite direction. We assume the object to be a closed, geometrically smooth object, thus, we can determine the surface normal at the occluding boundary. By propagating the determination of $\phi$ from the occluding boundary to the inside part of the object region, we can solve this $\phi$-ambiguity easily.

Zenith angle can be determined by DOP(degree of polarization). DOP represents how much the light has been polarized. DOP is 1 for perfectly polarized light, 0 for unpolarized light, and it varies between 0 to 1 . The definition of DOP is:

$$
\rho=\frac{I_{\max }-I_{\min }}{I_{\max }+I_{\min }}
$$

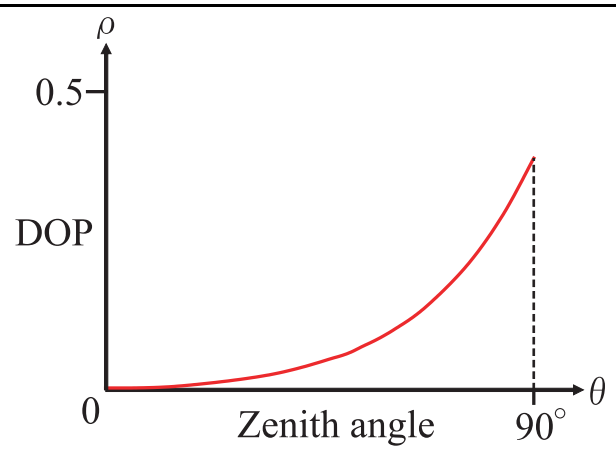

Figure 1. DOP(degree of polarization) of diffuse light $(n=1.7)$

DOP is related to zenith angle by following Equation $[18,19]$ :

$$
\rho=\frac{(n-1 / n)^{2} \sin ^{2} \theta}{2+2 n^{2}-(n+1 / n)^{2} \sin ^{2} \theta+4 \cos \theta \sqrt{n^{2}-\sin ^{2} \theta}}
$$

where $\rho$ is the DOP of diffuse light, $\theta$ is the zenith angle, and $n$ is the refractive index of the object to that of the air. Figure 1 represents this function. This function indicates that we can calculate the zenith angle by measuring the DOP of diffuse light. However, the function represents the DOP of an optically smooth surface. DOP of rough surface is smaller than that of smooth surface. Moreover, we have to know the refractive index. One solution to this problem is described in Section 4.3.

\section{Modeling photometrical and environmental information}

\subsection{Estimating illumination direction}

The direction of the light source can be represented as a polar coordinate system, since we do not intend to estimate the distance between the light source and the object, assuming that the light sources are sufficiently far from the object. Also, we assume that each light sources have the same radiant power. We first detect the brightest intensity of the specular component image. Then, from the surface normal $(\theta, \phi)$ of the detected point, we determine the direction of the light source as $(2 \theta, \phi)$ since we defined the camera direction as $\theta=0^{\circ}$. If there are many candidates of the direction of the light source, we manually choose what is considered to be correct.

\subsection{Estimating reflection parameters}

In this paper, we use simplified and discretized TorranceSparrow model [15] for the reflection model:

$$
\mathbf{I}=\mathbf{I}_{d}+\mathbf{I}_{s}
$$




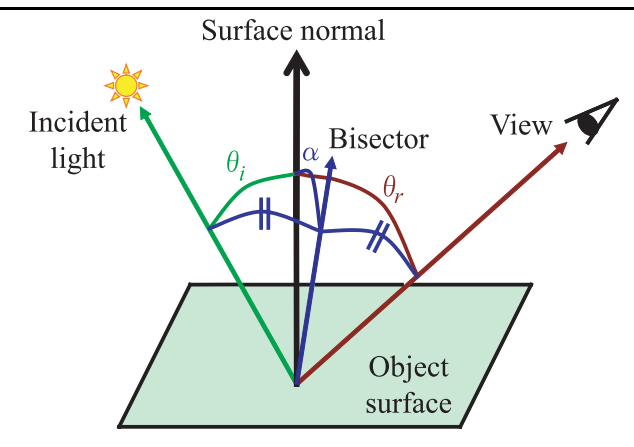

Figure 2. Geometrical location of surface normal, illumination direction, and viewing direction

$$
\begin{aligned}
\mathbf{I}_{d} & =\mathbf{K}_{d} \sum_{l=1}^{L} \cos \theta_{i} \\
\mathbf{I}_{s} & =\frac{\mathbf{K}_{s}}{\cos \theta_{r}} \sum_{l=1}^{L} \exp \left(-\frac{\alpha^{2}}{2 \sigma^{2}}\right)
\end{aligned}
$$

where $\mathbf{I}$ is the observed intensity, $\mathbf{I}_{d}$ is the diffuse reflection intensity, $\mathbf{I}_{s}$ is the specular reflection intensity, $\mathbf{K}_{d}$ is the diffuse reflection scale, $\theta_{i}$ is the angle between the incident light and the surface normal, $\mathbf{K}_{s}$ is the specular reflection scale, $\theta_{r}$ is the angle between the surface normal and the viewing direction, $\alpha$ is the angle between the surface normal and the bisector of incident light direction and the viewing direction, $\sigma$ is the surface roughness paramter, $L$ is a number of light sources, and $l=\{1, \ldots, L\}$ is a set of light sources(Figure 2).

Extracting illumination intensity from reflection scale (= $\mathbf{K}_{d}$ and $\mathbf{K}_{s}$ ) is an ill-posed problem in our framework, thus, we only estimate the reflection scale. For reflection parameters estimation, we assume that the Fresnel coefficient and the geometrical attenuation factor are constant. In this paper we use RGB color camera, thus, $\mathbf{I}, \mathbf{I}_{d}, \mathbf{I}_{s}, \mathbf{K}_{d}$, and $\mathbf{K}_{s}$ are 3-dimensional vectors.

We obtain $\mathbf{I}$ from the camera, $\mathbf{I}_{d}$ and $\mathbf{I}_{s}$ from the separated reflection components, $\theta_{i}, \theta_{r}$, and $\alpha$ from surface normal and illumination direction, and $l=\{1, \ldots, L\}$ from the procedure described in Section 3.1. Then, the remaining unknown parameter we intend to estimate are the diffuse reflectance $\mathbf{K}_{d}$, the specular reflectance $\mathbf{K}_{s}$, and surface roughness $\sigma$.

Diffuse reflectance is sometimes called a texture. By using surface normal and illumination distribution, we can calculate the diffuse reflectance $\mathbf{K}_{d}$ by Equation (4). We assume that all of surface points have the same $\mathbf{K}_{s}$ and $\sigma . \mathbf{K}_{s}$ and $\sigma$ can be estimated with non-linear least square method by minimizing the squared difference between the lefthand side and righthand side of Equation (5).

\section{Implementation}

\subsection{Specular Free Image}

Tan et al. [16] introduced a specular-free image in their method to separate an input image into diffuse component image and specular component image. Specular-free image is an image that is free from highlights but has different surface color from the input image. To produce the specular-free image, they proposed a two dimensional space, max.chromaticity-intensity space. In fact, the specular-free image can be also calculated by using other spaces such us a color space called $\mathbf{S}$ space defined by Bajcsy et al. [24]. In this paper, we use the following color space similar to $\mathbf{S}$ space:

$$
\begin{aligned}
& \mathbf{y}=\mathbf{A} \mathbf{x} \\
& \mathbf{A}=\left(\begin{array}{ccc}
1 & -\frac{1}{2} & -\frac{1}{2} \\
0 & \frac{\sqrt{3}}{2} & -\frac{\sqrt{3}}{2} \\
\frac{1}{3} & \frac{1}{3} & \frac{1}{3}
\end{array}\right) \\
& \mathbf{y}=\left[m_{1}, m_{2}, m_{3}\right]^{T}, \quad \mathbf{x}=[r, g, b]^{T}
\end{aligned}
$$

where $\mathbf{x}$ denotes the color in RGB space and $\mathbf{y}$ denotes the color in our proposed color space. Hue, saturation, and intensity can be defined as follows by using the parameters of $\left(m_{1}, m_{2}, m_{3}\right)$ space:

$$
\begin{aligned}
\text { Hue } & =\arctan \frac{m_{2}}{m_{1}} \\
\text { Saturation } & =\sqrt{m_{1}^{2}+m_{2}^{2}} \\
\text { Intensity } & =m_{3}
\end{aligned}
$$

For the normalized image (= image that has pure-white specular component), we can easily obtain the specular-free image by substituting the saturation into the intensity:

$$
\left(m_{1}^{\mathrm{sf}}, m_{2}^{\mathrm{sf}}, m_{3}^{\mathrm{sf}}\right) \leftarrow\left(m_{1}, m_{2}, a \sqrt{m_{1}^{2}+m_{2}^{2}}\right)
$$

where $a$ is an arbitrary value and $\left(m_{1}^{\text {sf }}, m_{2}^{\text {sf }}, m_{3}^{\text {sf }}\right)$ represents the specular free image.

Under the definition of hue, saturation, and intensity presented in this paper, this specular-free image will have the same hue and saturation but different intensity to the actual diffuse component image. However, the image is useful, since calculating the polarization data can be done from it. Unlike diffuse component image which is in certain circumstances more difficult to obtain, the specular-free image is obtainable using one-pixel-based operation and almost real time.

Note that DOP is defined only by the ratio of the intensity (Equation (1)) not the absolute value of the intensity, thus, the DOP calculated from specular-free image will be just the same as that calculated from diffuse component image.

\subsection{Unpolarized World Assumption}

Polarization data is sometimes affected by surrounding ambient light. To cancel out this interference, we propose 
an algorithm called Unpolarized-World algorithm by using unpolarized-world assumption. By assuming the reflected light as an unpolarized light, Unpolarized-World algorithm easily estimates the amibient polarized light caused by surrounding objects. Note that we observe only diffuse reflection for calculating polarization data. DOP is very low for diffuse reflection and is much lower for a rough surface, thus, the observed diffuse light is almost unpolarized.

Polarization state of any light can be expressed by Stokes vector [25]. Original Stokes vector is a $4 \mathrm{D}$ vector, however, we do not consider any circular polarization, thus, we can represent the light by $3 \mathrm{D}$ vector ignoring the fourth parameter of Stokes vector. Our Unpolarized-World algorithm modifies the input Stokes vector as:

$$
\tilde{\mathbf{I}}=\mathbf{I}+\mathbf{s}
$$

where $\mathbf{I}, \mathbf{s}$, and $\tilde{\mathbf{I}}$ is $3 \mathrm{D}$ Stokes vector, and represent the input light, ambient light, and modified light, respectively. $\mathbf{I}$ and $\mathbf{s}$ are represented as:

$$
\begin{aligned}
& \mathbf{I}=\left(\begin{array}{ccc}
1 & 0 & 0 \\
0 & \cos 2 \phi & -\sin 2 \phi \\
0 & \sin 2 \phi & \cos 2 \phi
\end{array}\right)\left(\begin{array}{c}
I_{\max }+I_{\min } \\
I_{\max }-I_{\min } \\
0
\end{array}\right) \\
& \mathbf{s}=\mu\left(\begin{array}{ccc}
1 & 0 & 0 \\
0 & \cos 2 \psi & -\sin 2 \psi \\
0 & \sin 2 \psi & \cos 2 \psi
\end{array}\right)\left(\begin{array}{l}
1 \\
1 \\
0
\end{array}\right)
\end{aligned}
$$

where $\mu$ denotes the intensity of ambient light, and $\psi$ is the polarization angle of ambient light. Thus, by substituting Equation (14) and (15) into Equation (13), we obtain:

$$
\tilde{\mathbf{I}}=\left(\begin{array}{c}
I_{\max }+I_{\min }+\mu \\
\left(I_{\max }-I_{\min }\right) \cos 2 \phi+\mu \cos 2 \psi \\
\left(I_{\max }-I_{\min }\right) \sin 2 \phi+\mu \sin 2 \psi
\end{array}\right)
$$

I is the input light, which is affected by ambient perfectly polarized light. We intend to obtain an unpolarized light $\tilde{\mathbf{I}}$ by adding a perfectly polarized light $\mathrm{s}$ to input light $\mathbf{I}$. Thus, the purpose is to estimate $\mu$ and $\psi$ to make the modified light $\tilde{\mathbf{I}}$ into an unpolarized light $\left(I_{\max }+I_{\min }+\mu\right)\left[\begin{array}{lll}1 & 0 & 0\end{array}\right]^{T}$. $\mu$ and $\psi$ can be determined by solving the least square problem. $\psi$ can be determined as:

$$
\psi=\frac{1}{2} \arctan \frac{\sum w\left(I_{\max }-I_{\min }\right) \sin 2 \phi}{\sum w\left(I_{\max }-I_{\min }\right) \cos 2 \phi}
$$

and $\mu$ can be determined as one of the two equations below:

$$
\begin{aligned}
& \mu=-\frac{\sum w\left(I_{\max }-I_{\min }\right) \cos 2 \phi}{\cos 2 \psi \sum w} \\
& \mu=-\frac{\sum w\left(I_{\max }-I_{\min }\right) \sin 2 \phi}{\sin 2 \psi \sum w}
\end{aligned}
$$

where $w$ is a weighting function. In this paper, we use $w=1-\rho$. If DOP $\rho$ is small, then weight $w$ is set to be large, meaning that if the light is almost unpolarized, then the weighting factor is set to be large.

DOP $\tilde{\rho}$ and phase angle $\tilde{\phi}$ can be calculated by the estimated parameters $\mu$ and $\psi$ as [25]:

$\tilde{\rho}=$

$$
\begin{aligned}
& \frac{\sqrt{\left(I_{\max }-I_{\min }\right)^{2}+2 \mu\left(I_{\max }-I_{\min }\right) \cos 2(\phi-\psi)+\mu^{2}}}{I_{\max }+I_{\min }+\mu} \\
& \tilde{\phi}=\frac{1}{2} \arctan \frac{\left(I_{\max }-I_{\min }\right) \sin 2 \phi+\mu \sin 2 \psi}{\left(I_{\max }-I_{\min }\right) \cos 2 \phi+\mu \cos 2 \psi}
\end{aligned}
$$

\subsection{Histogram Modification}

DOP depends on refractive index (Equation (2)). In addition, Equation (2) is derived by assuming the surface as an optically smooth surface. Rough surface causes depolarization and lowers the obtained DOP. Thus, the calculated zenith angle $\theta$ from Equation (2) by giving arbitrary refractive index will be different from the true value; we have to modify the calculated zenith angle $\theta$ to better represent the true zenith angle.

Estimating all these three unknown parameters (= zenith angle, refractive index, and surface roughness) is an illposed problem for a single polarization image. We took a statistical approach for modifying the zenith angle. Assume that we know the histogram of zenith angle calculated all over the surface. Then, by deforming the histogram of obtained zenith angle to the expected histogram, the zenith angle will represent the true value. In this paper, we simply used the histogram of hemisphere for the true histogram.

Histogram of hemisphere will be $2 N \sin \theta\left(0^{\circ} \leq \theta \leq\right.$ $90^{\circ}$ ), where $N$ is the number of surface points. The zenith angle can be modified as follows. First, we sort all the surface points in ascent order with respect to zenith angle. Then, calculate $\arcsin \sqrt{i / N}$ for the zenith angle of the $i$ th point of the sorted list.

\section{Measurement results}

First, we analyzed the accuracy of the polarization data by measuring a specular green hemisphere. For anlyzing the accuracy of this object, we did not apply any smoothing operations to the obtained data, however, the modification method described in Section 4.2 and 4.3 is applied to the obtained data. The average error of surface normal was $19^{\circ}$ for this green hemisphere; the average error is calculated by the average of the angle between the estimated surface normal and the true surface normal. According to this result, the input data seem to be less accurate, thus, for the subsequent measurements, we properly smoothed the input data to obtain a better estimation.

Next, we measure a yellow pear object shown in Figure 3(a). By the color-based separation, we separated the input image into specular and diffuse component images. From the polarizer-mounted camera, we obtained the DOP data and $\phi$ data from the specular-free image. Then, we calculated the surface normal of the object and integrated them into the height of the object(Figure 3(b)). 


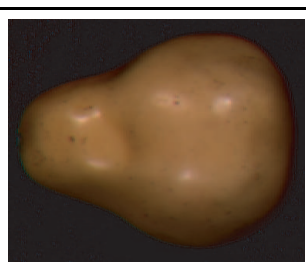

(a)

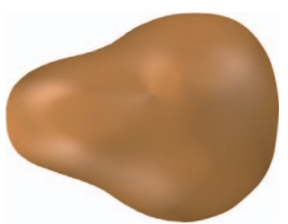

(c)

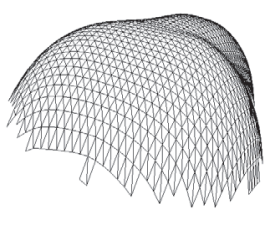

(b)

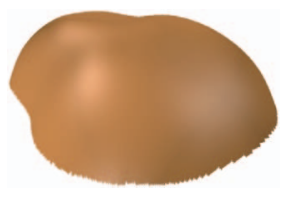

(d)
Figure 3. Result of pear: (a) True image, (b) Surface mesh, (c) Synthesized image, (d) Rendered from a novel view under different illumination

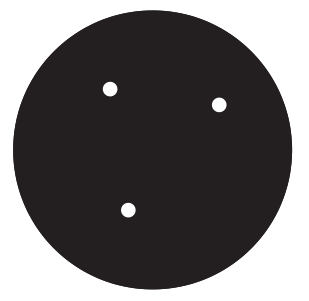

(a)

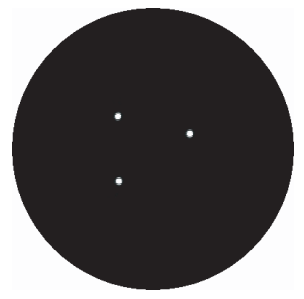

(b)
Figure 4. Illumination of pear: (a) Estimated illumination, (b) True illumination

By detecting the brightest intensity in specular component image, we determined the direction of light sources, which is depicted in Figure 4(a). For comparison, the true illumination distribution is shown in Figure 4(b). Estimated texture $\mathbf{K}_{d}$ was $(66,37,15)$. For the measurement results of the pear, we assumed that $\mathbf{K}_{d}$ is the same for all surface points. We estimated the reflection parameters, and resulted surface roughness $\sigma$ and specular scale $\mathbf{K}_{s}$ was 0.17 and 22 , respectively. Note that we normalized the input images to make the specular reflection component become white, thus, all three elements of $\mathbf{K}_{s}$ have the same value.

Rendered examples are shown in Figure 3(c) and (d). Figure 3(c) is rendered under the estimated parameters and illumination, which should be the same as Figure 3(a). Figure 3(d) is an another example which is rendered under the different object direction and the different illumination position.

Figure 5(a) is a real image of pink dinosaur object to measure. Estimated shape is depicted in Figure 5(b) and

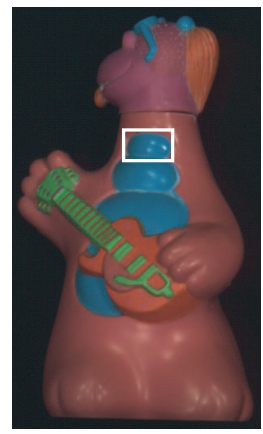

(a) (b)

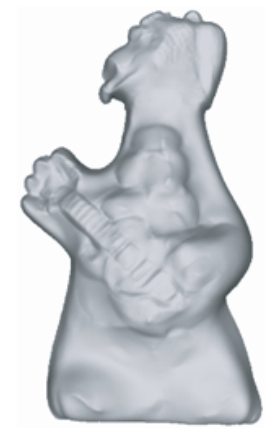

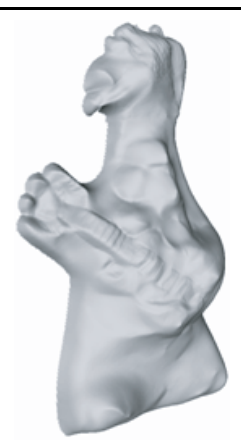

(c)
Figure 5. (a) Real image of dinosaur: Illumination distribution is estimated by analyzing the white region, (b)(c) Estimated shape of dinosaur.
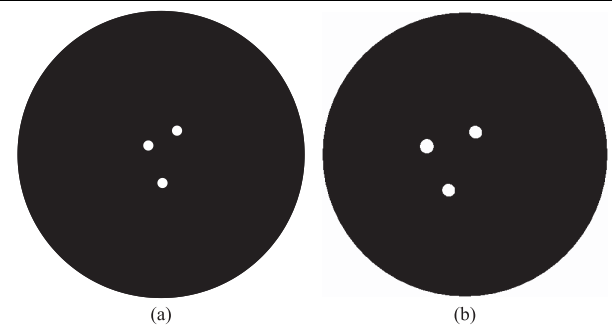

Figure 6. Illumination of dinosaur: (a) Estimated illumination, (b) True illumination

(c). Figure 6(a) is the estimated illumination and Figure 6(b) is the ground truth. We only used the area bounded by white box in Figure 5(a) to estimate the illumination distribution. This area is smoothed enough to obtain a better estimation. Error for illumination direction was $21^{\circ}, 4.4^{\circ}$, and $15^{\circ}$ for northwest light source, northeast light source, and south light source, respectively (Here, "north" is represented as the upper direction in Figure 6). Estimated surface roughtness $\sigma$ and specular scale $\mathbf{K}_{s}$ was 0.071 and 93, respectively. Estimated diffuse scale $\mathbf{K}_{d}$ is shown in Figure 7(a). Figure 7(b) is rendered under the estimated parameters and illumination, which should be the same as Figure 5(a). Figure 7(c) is an another example which is rendered under the different object direction and the different illumination position. Appearance difference between these rendered images and the real image tells us that the specular factors, $\mathbf{K}_{s}$ and $\sigma$, seem to be overestimated. To improve the accuracy of the estimation method lies for our future work. 


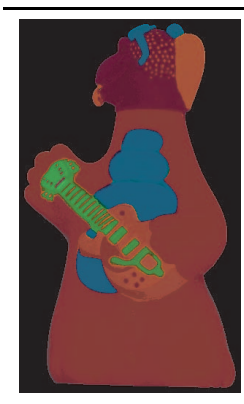

(a)

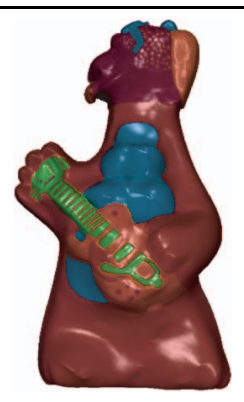

(b)

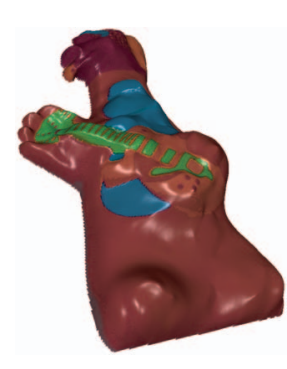

(c)
Figure 7. (a) Diffuse reflection scale $\mathbf{K}_{d}$, (b) Rendered image, (c) Rendered under different viewpoint

\section{Conclusion}

We have introduced a method to estimate shape, texture, surface roughness, and illumination distribution from a single view in one integrated framework. Our method estimates the direction of multiple light sources, without requiring any special light sources such as laser beam nor structured pattern light. One of our main contributions is the improvement of the shape-from-polarization technique. We successfully obtained the shape of the object from a single view by analyzing the polarization effect of the light, and demonstrated the ability of our method to determine the shape of objects using real images. We also estimated the reflection parameters and illumination distribution to confirm that the estimated shape is enough precise to be applied to another process.

\section{References}

[1] Y. Sato, K. Ikeuchi, "Temporal-color space analysis of reflection," J. Opt. Soc. Am. A, 11(11), pp.2990-3002, 1994.

[2] S. Tominaga, N. Tanaka, "Estimating Reflection Parameters from a Single Color Image," IEEE Computer Graphics and Applications, 20(5), pp.58-66, 2000.

[3] Q. Zheng, R. Chellapa, "Estimation of Illuminant Direction, Albedo, and Shape from Shading," IEEE Trans. Patt. Anal. Mach. Intell., 13(7), pp.680-702, 1991.

[4] S.K. Nayar, X.S. Fang, T. Boult, "Separation of Reflection Components Using Color and Polarization," IEEE Intl. J. Computer Vision, 21(3), pp.163-186, 1996.

[5] C-Y. Kim, A.P. Petrov, H-K. Choh, Y-S. Seo, I-S. Kweon, "Illuminant direction and shape of a bump," J. Opt. Soc. Am. A, 15(9), pp.2341-2350, 1998.

[6] K. Ikeuchi, K. Sato, "Determining reflectance properties of an object using range and brightness images," IEEE Trans. Patt. Anal. Mach. Intell., 13(11), pp.1139-1153, 1991.

[7] I. Sato, Y. Sato, K. Ikeuchi, "Illumination distribution from brightness in shadows: adaptive estimation of illumintion distribution with unknown reflectance properties in shadow re- gions," Proc. Intl. Conf. Computer Vision, pp.875-882, 1999.

[8] R. Ramamoorthi, P. Hanrahan, "A signal processing framework for inverse rendering," Proc. SIGGRAPH 2001, pp.379$387,2001$.

[9] K. Nishino, Z. Zhang, K. Ikeuchi, "Determining Reflectance Parameters and Illumination Distribution from a Sparse Set of Images for View-dependent Image Synthesis," Proc. IEEE Intl. Conf. Computer Vision, pp.599-606, 2001.

[10] K. Hara, K. Nishino, A. Nakazawa, K. Ikeuchi, "Estimating Light Position and Surface Reflectance from Specular Reflection under Perspective Projection," Proc. IAPR Workshop on Machine Vision Applications, pp.566-571, 2002.

[11] K. Hara, K. Nishino, K. Ikeuchi, "Determining Reflectance and Light Position from a Single Image Without Distant Illumination Assumption," Proc. IEEE Intl. Conf. Computer Vision, 2003. to appear

[12] S. Rahmann, "Inferring 3D scene structure from a single polarization image," SPIE Proc. 3826: Conf. Polarization and Color Techniques in Industrial Inspection, pp.22-33, 1999.

[13] B.K.P. Horn, Robot vision, MIT Press, 1986.

[14] K. Ikeuchi, "Reconstructing a depth map from intensity maps," Proc. Intl. Conf. Pattern Recognition, pp.736-738, 1984.

[15] K.E. Torrance, E.M. Sparrow, "Theory for off-specular reflection from roughened surfaces," J. Opt. Soc. Am., 57(9), pp.1105-1114, 1967.

[16] R.T. Tan, K. Ikeuchi, "Separating Reflection Components of Textured Surface using a Single Image," Proc. IEEE Intl. Conf. Computer Vision, 2003. to appear

[17] R.T. Tan, K. Nishino, K. Ikeuchi, "Illumination Chromaticity Estimation using Inverse-Intensity Chromaticity Space," Proc. IEEE Conf. Computer Vision and Pattern Recognition, pp.I:673-680, 2003.

[18] M. Born, E. Wolf, Principles of optics, Pergamon, 1959.

[19] L.B. Wolff, T.E. Boult, "Constraining object features using a polarization reflectance model," IEEE Trans. Patt. Anal. Mach. Intell., 13(7), pp.635-657, 1991.

[20] M. Saito, Y. Sato, K. Ikeuchi, H. Kashiwagi, "Measurement of surface orientations of transparent objects by use of polarization in highlight," J. Opt. Soc. Am. A, 16(9), pp.2286-2293, 1999.

[21] S. Rahmann, N. Canterakis, "Reconstruction of specular surfaces using polarization imaging," Proc. IEEE Conf. Computer Vision and Pattern Recognition, pp.I:149-155, 2001.

[22] D. Miyazaki, M. Saito, Y. Sato, K. Ikeuchi, "Determining surface orientations of transparent objects based on polarization degrees in visible and infrared wavelengths," J. Opt. Soc. Am. A, 19(4), pp.687-694, 2002.

[23] D. Miyazaki, M. Kagesawa, K. Ikeuchi, "Polarization-based Transparent Surface Modeling from Two Views," Proc. IEEE Intl. Conf. Computer Vision, 2003. to appear

[24] R. Bajcsy, S.W. Lee, A. Leonardis, "Detection of diffuse and specular interface reflections by color image segmentation," Intl. J. Computer Vision, 17(3), pp.249-272, 1996.

[25] W.A. Shurcliff, Polarized light: production and use, Harvard University Press, 1962. 